\title{
Theoretical Study of Oxozirconium Porphyrin, Octamethylporphyrin, Porphyrazine and Phthalocyanine Complexes
}

\author{
Alexander V. Zakharov \\ Research Institute of Chemistry of Macroheterocyclic Compounds, Ivanovo State University of Chemistry and Technology, \\ 153000 Ivanovo, Russian Federation \\ E-mail: Alexander.V.Zakharov@gmail.com.
}

\begin{abstract}
The molecular structures, vibrational spectra and electronic spectra of oxozirconium porphyrin, octamethylporphyrin, porphyrazine and phthalocyanine have been studied by density functional theory and time-dependent density functional theory calculations utilizing B3LYP method and triple- $\zeta$ valence basis sets.
\end{abstract}

Keywords: Zirconium, porphyrin, phthalocyanine, porphyrazine, density functional theory, molecular structure, vibrational spectra.

\section{Introduction}

Among porphyrin and phthalocyanine complexes of IV period metals the titanium complexes are most widely studied. Oxotitanum phthalocyanine (TiOPc) is a wellknown near-IR photoconductor which is applied in GaAsAl laser printers, and it was studied extensively. Its structures in crystals ${ }^{[1]}$ as well as in the gas phase ${ }^{[2]}$ were investigated experimentally, by X-ray crystallography and gas-phase electron diffraction, respectively. Electronic spectra, photoconductivity and optical properties of the solid state TiOPc were also intensively studied (for detailed list of references see $\left.{ }^{[2,3]}\right)$.

Until recently, the information about the structure of oxotitanium porphyrins was absent. In 2006 Sakurai et $a l^{[4]}$ investigated a discoloration reaction of oxotitanium porphyrin (TiOP) by density functional theory calculations. According to the data of the study, ${ }^{[4]}$ geometry optimization yielded a structure of $C_{4 \mathrm{v}}$ symmetry for TiOP. In 2008 a study of molecular structures, vibrational spectra and electronic spectra of oxotitanium complexes of porphyrin (TiOP), octamethylporphyrin (TiO(OMP)), porphyrazine ( $\mathrm{TiOPz})$ and phthalocyanine ( $\mathrm{TiOPc}$ ) by density functional theory computations was carried out. ${ }^{[3]}$ All four molecules were found to possess the equilibrium structures of $C_{4 \mathrm{v}}$ symmetry with convex macrocycles. ${ }^{[3]}$

On the other hand, information about structures of zirconium porphyrin and phthalocyanine complexes is scant. Structural studies of oxozirconium complexes are absent. X-ray crystallographic studies of other zirconium complexes with axial substituents exist, namely of chlorocis-bis(trimethylsilyl)amido (5,10,15,20-tetraphenyl-porphyrinato)zirconium(IV), ${ }^{[5]}$ dichloro-octaethylporphyrinatozirconium(IV) ${ }^{[6]}$ bis(tert-butoxy)octaethylporphyrinatozirco nium(IV) ${ }^{[6]}$ dimethyloctaethylporphyrinatozirconium(IV) ${ }^{[6]}$ (octaethylporphyrinato)trimethylsilylmethylzirconium(IV) ${ }^{[7]}$ and bis(trimethylsilylmethyl)octaethylporphyrinzirconium. ${ }^{[8]}$ All the complexes mentioned feature the zirconium atom displaced out of the average plane of four nitrogen atoms, a convex macrocycle and the cis-configuration of extraligands, except the (octaethylporphyrinato)trimethylsilylmethylzirconium, where only one axial substituent is present.

The present study aims at extending the knowledge of $\mathrm{Zr}$ complexes with macrohetrocyclic ligands as well as at comparison with similar well-studied TiO coordination compounds.

\section{Computational}

The molecules of oxozirconium complexes have been studied by density functional theory (DFT) computations utilizing the B3LYP hybrid method (Becke + Slater + HF exchange and LYP + VWN5 correlation). All geometry optimization and force field calculations were performed using PC GAMESS 7.0 QC package, ${ }^{[9]}$ which is partially based on the GAMESS (US) software package, ${ }^{[10]}$ running under Linux operating system.

The core electron shells $(1 \mathrm{~s} 2 \mathrm{~s} 2 \mathrm{p} 3 \mathrm{~s} 3 \mathrm{p} 3 \mathrm{~d})$ of the zirconium atom were described by a relativistic effective core potential (ECP). ${ }^{[1]}$ For description of the zirconium valence shells a $(8 \mathrm{~s} 7 \mathrm{p} 6 \mathrm{~d} 2 \mathrm{f} 1 \mathrm{~g} / 6 \mathrm{~s} 5 \mathrm{p} 3 \mathrm{~d} 2 \mathrm{f} 1 \mathrm{~g})$ basis set ${ }^{[11,12]}$ has been used. All other atoms $(\mathrm{C}, \mathrm{N}, \mathrm{O}, \mathrm{H})$ were described by triple- $\zeta$ valence basis sets $(10 \mathrm{~s} 6 \mathrm{p} / 5 \mathrm{~s} 3 \mathrm{p})$ in case of $\mathrm{C}, \mathrm{N}, \mathrm{O}$ and $(5 \mathrm{~s} / 3 \mathrm{~s})$ in case of $\mathrm{H}^{[13]}$ (designated TZV in GAMESS) with addition of polarization functions $\left(\zeta_{d}\right.$ $=1.28(\mathrm{O}), \zeta_{\mathrm{d}}=0.98(\mathrm{~N}), \zeta_{\mathrm{d}}=0.72^{[14]}$ and $\left.\zeta_{\mathrm{p}}=1.0(\mathrm{H})^{[15]}\right)$. Such level of theory has been found to yield accurate structural parameters in earlier combined (experimental and theoretical) studies of oxotitanium phthalocyanine ${ }^{[2]}$ and other large macroheterocyclic molecules. ${ }^{[16-20]}$

Singlet and triplet excited states and the electronic spectra were studied using time-dependent density functional theory (TDDFT) implemented in PC GAMESS 7.0. ${ }^{[9]}$ In all four molecules the molecular orbitals formed mostly by $1 s$ orbitals of $\mathrm{C}, \mathrm{N}$, and $\mathrm{O}$ atoms, namely 25 first (lowest energy) orbitals in oxozirconium porphyrin and porphyrazine, 33 in octamethylporphyrin and 41 in phthalocyanine, were excluded ("frozen") from the TDDFT calculations.

Visualization of the results has been done using the MacMolPlt software. ${ }^{[21]}$ Supplementary material is available at http://dx.doi.org/10.6060/mhc2012.120257z 


\section{Results and Discussion}

According to the results of our calculations all four molecules have the equilibrium structures of $C_{4 \mathrm{v}}$ symmetry, shown in Figures 1-4. The structural parameters (bond distances, valence angles and dihedral angles significantly differ from either 0 or 180 degrees) of the molecules in question are presented in Table 1; data from study ${ }^{[3]}$ of oxotitanium complexes are given for comparison.

The structural parameters of the macroheterocyclic ligands remained almost unchanged compared to the oxotitanium complexes, with two noticeable exceptions. In porphyrin and octamethylporphyrin oxozirconium complexes the distances between $\mathrm{C}_{\mathrm{p} 1}$ atoms in the pyrrole rings and $\mathrm{C}_{\mathrm{m}}$ (meso-atoms) are longer than the corresponding distances in similar oxotitanium complexes by 0.008 A. Porphyrazine and phthalocyanine complexes feature similar elongations of $\mathrm{r}\left(\mathrm{C}_{\mathrm{pl}}-\mathrm{N}_{\mathrm{m}}\right)$ distances, but to a lesser extent $(0.004-0.005$ $\AA)$. The other differences are in the values of $\alpha\left(\mathrm{N}-\mathrm{C}_{\mathrm{p} 1}-\mathrm{C}_{\mathrm{p} 2}\right)$ valence angles; they are smaller by 0.7 degrees in $\mathrm{ZrOP}$ and

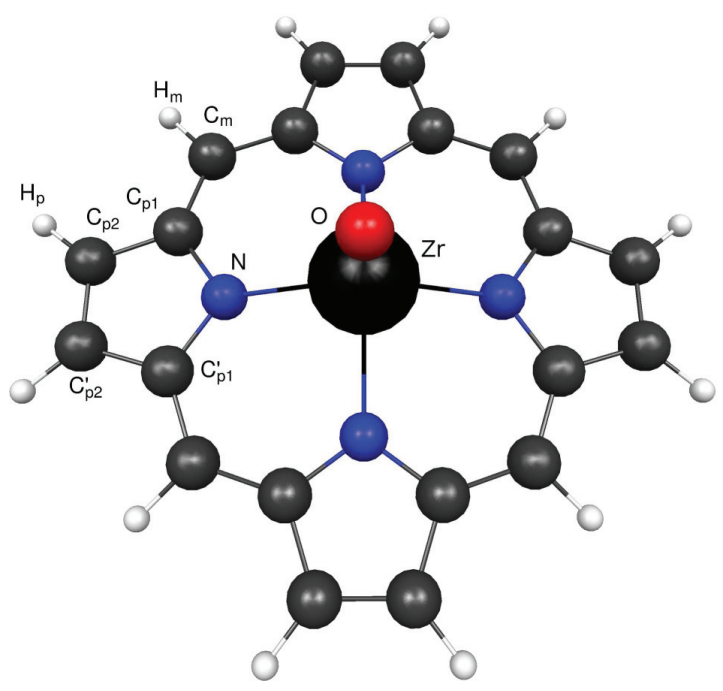

Figure 1. Molecular structure of oxozirconium porphyrin.

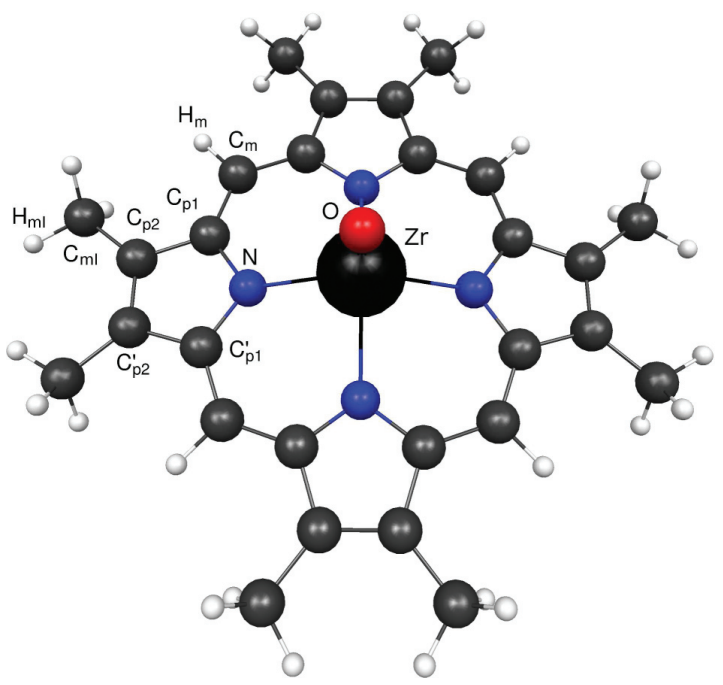

Figure 2. Molecular structure of oxozirconium octamethylporphyrin.
$\mathrm{ZrO}(\mathrm{OMP})$ compared to TiOP and TiO(OMP) and by $0.3-0.5$ degrees in $\mathrm{ZrOPz}$ and $\mathrm{ZrOPc}$ compared to TiOPz and TiOPc. The changes of $\mathrm{r}\left(\mathrm{C}_{\mathrm{p} 1}-\mathrm{C}_{\mathrm{m}}\right) / \mathrm{r}\left(\mathrm{C}_{\mathrm{pl}}-\mathrm{N}_{\mathrm{m}}\right)$ distances and $\alpha\left(\mathrm{N}-\mathrm{C}_{\mathrm{p} 1}-\right.$ $\mathrm{C}_{\mathrm{p} 2}$ ) valence angles are clearly interdependent. The described changes result in a significant increase of the size of the coordination cavity. Thus, the $\mathrm{N} \cdots \mathrm{N}$ distance (i.e. the side of the square formed by four central nitrogen atoms) increased by $0.063 \AA$ in porphyrins, from $2.892-2.893 \AA$ in TiOP and TiO(OMP) to 2.955-2.956 $\AA$ in $\mathrm{ZrOP}$ and $\mathrm{ZrO}(\mathrm{OMP})$. The change in phthalocyanine and porphyrazine complexes is slightly smaller, $0.057 \AA$ (from 2.805 to $2.862 \AA$ in phthalocyanine and from 2.766 to $2.823 \AA$ in porphyrazine).

This widening of the coordination cavity is certainly caused by a larger size of the $\mathrm{Zr}^{4+}$ ion with ionic radius of $0.72 \AA$, compared to $0.60 \AA$ of $\mathrm{Ti}^{4+}$. The increase of the ionic radius also results in significant heightening of the $\mathrm{ZrO}_{4}$ pyramid in comparison to $\mathrm{TiO}_{4}$, to $0.831 \AA$ in oxozirconium porphyrin, $0.836 \AA$ in octamethylporphyrin, $0.976 \AA$ in porphyrazine and $0.933 \AA$ in phthalocyanine complexes (the heights in oxotitanium complexes are 0.577 to $\left.0.690 \AA,{ }^{[3]}\right)$.

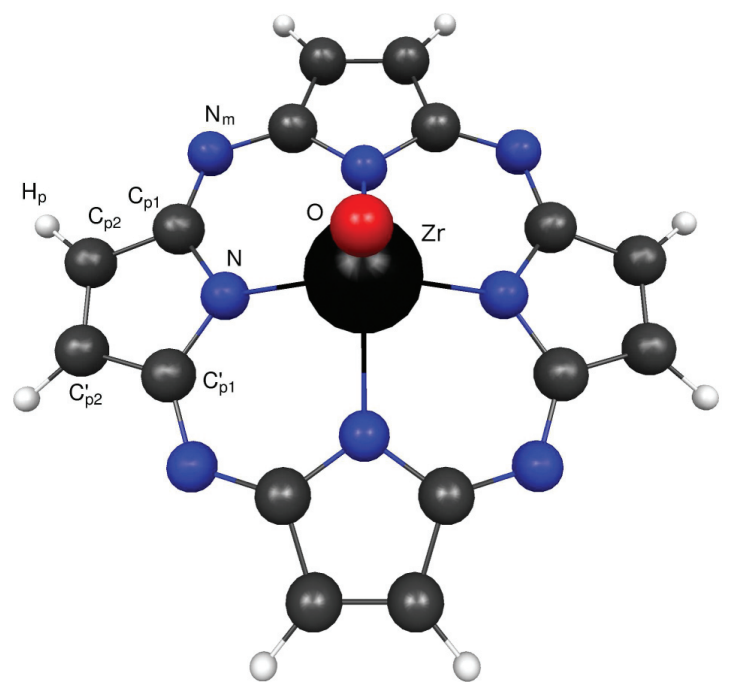

Figure 3. Molecular structure of oxozirconium porphyrazine.

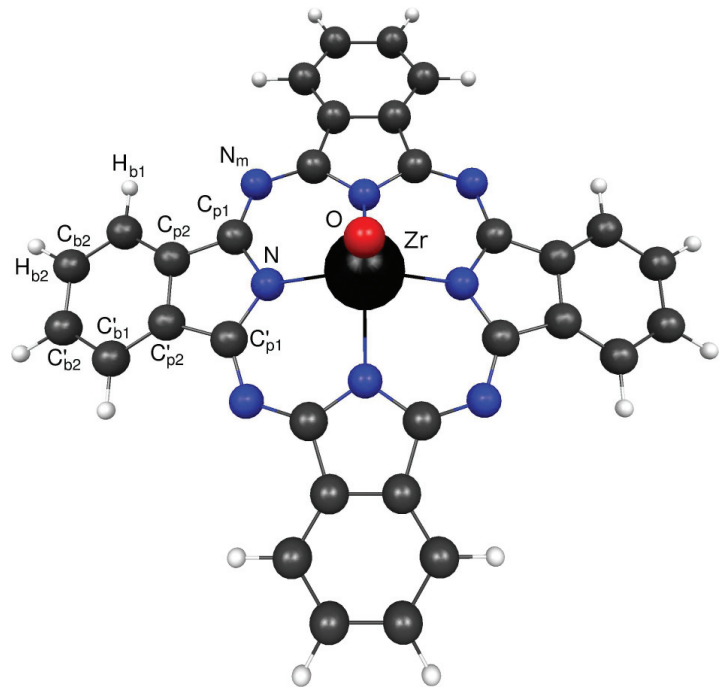

Figure 4. Molecular structure of oxozirconium phthalocyanine. 
Table 1. Structural parameters of oxozirconium and oxotitanium (data from study $\left.{ }^{[3]}\right)$ complexes $(\mathrm{P}$ - porphyrin, OMP - octamethylporphyrin, $\mathrm{Pz}$ - porphyrazine, $\mathrm{Pc}-$ phthalocyanine $)^{\mathrm{a}}$

\begin{tabular}{|c|c|c|c|c|c|c|c|c|}
\hline & \multicolumn{4}{|c|}{ Oxozirconium complexes } & \multicolumn{4}{|c|}{ Oxotitanium complexes ${ }^{[3]}$} \\
\hline & $\mathrm{P}$ & OMP & $\mathrm{Pz}$ & $\mathrm{Pc}$ & $\mathrm{P}$ & OMP & $\mathrm{Pz}$ & $\mathrm{Pc}$ \\
\hline \multicolumn{9}{|c|}{ Bond distances, $\AA$} \\
\hline $\mathrm{r}(\mathrm{Zr}-\mathrm{O})$ & 1.768 & 1.774 & 1.771 & 1.769 & 1.614 & 1.617 & 1.620 & 1.617 \\
\hline $\mathrm{r}(\mathrm{Zr}-\mathrm{N})$ & 2.249 & 2.251 & 2.222 & 2.229 & 2.125 & 2.125 & 2.074 & 2.087 \\
\hline $\mathrm{r}\left(\mathrm{N}-\mathrm{C}_{\mathrm{p} 1}\right)$ & 1.372 & 1.370 & 1.372 & 1.375 & 1.373 & 1.372 & 1.373 & 1.376 \\
\hline $\mathrm{r}\left(\mathrm{Cm}-\mathrm{C}_{\mathrm{p} 1}\right) / \mathrm{r}\left(\mathrm{N}_{\mathrm{m}}-\mathrm{C}_{\mathrm{p} 1}\right)^{\mathrm{b}}$ & 1.400 & 1.400 & 1.331 & 1.327 & 1.392 & 1.392 & 1.327 & 1.323 \\
\hline $\mathrm{r}\left(\mathrm{C}_{\mathrm{p} 1}-\mathrm{C}_{\mathrm{p} 2}\right)$ & 1.443 & 1.453 & 1.455 & 1.458 & 1.441 & 1.450 & 1.453 & 1.456 \\
\hline $\mathrm{r}\left(\mathrm{C}_{\mathrm{p} 2}-\mathrm{C}_{\mathrm{p} 2}\right)$ & 1.363 & 1.372 & 1.358 & 1.409 & 1.361 & 1.370 & 1.357 & 1.406 \\
\hline $\mathrm{r}\left(\mathrm{C}_{\mathrm{p} 2}-\mathrm{C}_{\mathrm{m} 1}\right)$ & & 1.497 & & & & 1.498 & & \\
\hline $\mathrm{r}\left(\mathrm{C}_{\mathrm{p} 2}-\mathrm{C}_{\mathrm{b} 1}\right)$ & & & & 1.393 & & & & 1.393 \\
\hline $\mathrm{r}\left(\mathrm{C}_{\mathrm{b} 1}-\mathrm{C}_{\mathrm{b} 2}\right)$ & & & & 1.391 & & & & 1.391 \\
\hline $\mathrm{r}\left(\mathrm{C}_{\mathrm{b} 2}-\mathrm{C}_{\mathrm{b} 2}^{\prime}\right)$ & & & & 1.405 & & & & 1.406 \\
\hline $\mathrm{r}\left(\mathrm{C}_{\mathrm{m}}-\mathrm{H}_{\mathrm{m}}\right)$ & 1.083 & 1.083 & & & 1.083 & 1.082 & & \\
\hline $\mathrm{r}\left(\mathrm{C}_{\mathrm{p} 2}-\mathrm{H}_{\mathrm{p}}\right)$ & 1.079 & & 1.078 & & 1.079 & & 1.078 & \\
\hline $\mathrm{r}\left(\mathrm{C}_{\mathrm{ml}}-\mathrm{H}_{\mathrm{ml}}\right)$ & & 1.090 & & & & 1.090 & & \\
\hline $\mathrm{r}\left(\mathrm{C}_{\mathrm{ml}}-\mathrm{H}_{\mathrm{ml}}^{\prime}\right), \mathrm{r}\left(\mathrm{C}_{\mathrm{ml}}-\mathrm{H}_{\mathrm{ml}}^{\prime \prime}\right)$ & & 1.094 & & & & 1.094 & & \\
\hline $\mathrm{r}\left(\mathrm{C}_{\mathrm{b} 1}-\mathrm{H}_{\mathrm{b} 1}\right)$ & & & & 1.082 & & & & 1.082 \\
\hline $\mathrm{r}\left(\mathrm{C}_{\mathrm{b} 2}-\mathrm{H}_{\mathrm{b} 2}\right)$ & & & & 1.083 & & & & 1.083 \\
\hline \multicolumn{9}{|c|}{ Valence angles, degrees } \\
\hline$\alpha(\mathrm{O}-\mathrm{Zr}-\mathrm{N})$ & 111.7 & 111.8 & 116.1 & 114.7 & 105.8 & 105.8 & 109.4 & 108.1 \\
\hline$\alpha\left(\mathrm{Zr}-\mathrm{N}-\mathrm{C}_{\mathrm{pl}}\right)$ & 125.0 & 125.0 & 125.3 & 124.3 & 125.6 & 125.7 & 125.4 & 124.6 \\
\hline$\alpha\left(\mathrm{N}-\mathrm{C}_{\mathrm{p} 1}-\mathrm{C}_{\mathrm{m}}\right)^{\mathrm{b}}$ & 125.2 & 124.9 & 127.4 & 127.5 & 125.2 & 124.9 & 127.3 & 127.5 \\
\hline$\alpha\left(\mathrm{N}-\mathrm{C}_{\mathrm{p} 1}-\mathrm{C}_{\mathrm{p} 2}\right)$ & 108.8 & 109.3 & 108.8 & 108.3 & 109.5 & 110.0 & 109.1 & 108.8 \\
\hline$\alpha\left(\mathrm{C}_{\mathrm{p} 1}-\mathrm{C}_{\mathrm{p} 2}-\mathrm{C}_{\mathrm{p} 2}\right)$ & 107.2 & 106.8 & 107.2 & 106.7 & 107.1 & 106.7 & 107.1 & 106.7 \\
\hline$\alpha\left(\mathrm{C}_{\mathrm{p} 1}-\mathrm{C}_{\mathrm{p} 2}-\mathrm{C}_{\mathrm{m}}\right)$ & & 124.6 & & & & 124.6 & & \\
\hline$\alpha\left(\mathrm{C}_{\mathrm{p} 1}-\mathrm{C}_{\mathrm{p} 2}-\mathrm{C}_{\mathrm{b} 1}\right)$ & & & & 132.2 & & & & 132.2 \\
\hline$\alpha\left(\mathrm{C}_{\mathrm{p} 1}-\mathrm{C}_{\mathrm{p} 2}-\mathrm{H}_{\mathrm{p}}\right)$ & 125.0 & & 123.8 & & 124.9 & & 123.7 & \\
\hline$\alpha\left(\mathrm{C}_{\mathrm{p} 2}-\mathrm{C}_{\mathrm{m} 1}-\mathrm{H}_{\mathrm{ml}}\right)$ & & 111.5 & & & & 111.4 & & \\
\hline$\alpha\left(\mathrm{C}_{\mathrm{p} 2}-\mathrm{C}_{\mathrm{ml}}-\mathrm{H}_{\mathrm{ml}}^{\prime}\right)$ & & 111.4 & & & & 111.5 & & \\
\hline$\alpha\left(\mathrm{C}_{\mathrm{p} 2}-\mathrm{C}_{\mathrm{ml}}-\mathrm{H}_{\mathrm{ml}}^{\prime \prime}\right)$ & & 111.6 & & & & 111.6 & & \\
\hline$\alpha\left(\mathrm{C}_{\mathrm{p} 2}-\mathrm{C}_{\mathrm{b} 1}-\mathrm{C}_{\mathrm{b} 2}\right)$ & & & & 117.8 & & & & 117.7 \\
\hline$\alpha\left(\mathrm{C}_{\mathrm{b} 1}-\mathrm{C}_{\mathrm{b} 2}-\mathrm{C}_{\mathrm{b} 2}^{\prime}\right)$ & & & & 121.2 & & & & 121.2 \\
\hline$\alpha\left(\mathrm{C}_{\mathrm{p} 2}-\mathrm{C}_{\mathrm{b} 1}-\mathrm{H}_{\mathrm{bl}}\right)$ & & & & 120.7 & & & & 120.7 \\
\hline$\alpha\left(\mathrm{C}_{\mathrm{b} 1}-\mathrm{C}_{\mathrm{b} 2}-\mathrm{H}_{\mathrm{b} 2}\right)$ & & & & 119.6 & & & & 119.6 \\
\hline \multicolumn{9}{|c|}{ Dihedral angles, degrees } \\
\hline$\gamma\left(\mathrm{O}-\mathrm{Zr}-\mathrm{N}-\mathrm{C}_{\mathrm{p} 1}\right)$ & -80.7 & -80.4 & -82.1 & -81.8 & 81.3 & 81.0 & 81.8 & 81.9 \\
\hline$\gamma\left(\mathrm{Zr}-\mathrm{N}-\mathrm{C}_{\mathrm{pl}}-\mathrm{C}_{\mathrm{m}}\right)^{\mathrm{b}}$ & -20.6 & -20.9 & -22.1 & -21.7 & 16.7 & 16.9 & 18.6 & 17.7 \\
\hline$\gamma\left(\mathrm{Zr}-\mathrm{N}-\mathrm{C}_{\mathrm{p} 1}-\mathrm{C}_{\mathrm{p} 2}\right)$ & 162.7 & 162.1 & 163.1 & 162.2 & -165.0 & -164.5 & -164.5 & -164.5 \\
\hline$\gamma\left(\mathrm{C}_{\mathrm{p} 2}-\mathrm{C}_{\mathrm{p} 2}^{\prime}-\mathrm{C}_{\mathrm{m} 1}-\mathrm{H}_{\mathrm{ml}}\right)$ & & 3.4 & & & & -2.0 & & \\
\hline$\gamma\left(\mathrm{C}_{\mathrm{p} 2}-\mathrm{C}_{\mathrm{p} 2}^{\prime}-\mathrm{C}_{\mathrm{ml}}-\mathrm{H}_{\mathrm{ml}}^{\prime}\right)$ & & 123.5 & & & & -122.0 & & \\
\hline$\gamma\left(\mathrm{C}_{\mathrm{p} 2}-\mathrm{C}_{\mathrm{p} 2}^{\prime}-\mathrm{C}_{\mathrm{m} 1}-\mathrm{H}_{\mathrm{ml}}^{\prime \prime}\right)$ & & 116.6 & & & & 118.0 & & \\
\hline
\end{tabular}

${ }^{a}$ The labeling of atoms used throughout the text and tables is the following (see also Figures 1-4): $\mathrm{N}-\mathrm{N}$ atoms in the pyrrole rings; $\mathrm{C}_{\mathrm{m}}$ and $\mathrm{N}_{\mathrm{m}}$ - meso (methine bridge) $\mathrm{C}$ atoms ( $\mathrm{ZrOP}, \mathrm{ZrO}(\mathrm{OMP})$ ) and meso $\mathrm{N}$ atoms ( $\mathrm{ZrOPz}, \mathrm{ZrOPc}$ ), respectively; $\mathrm{C}_{\mathrm{pl}}-\mathrm{C}$ atoms in the pyrrole rings bonded to $\mathrm{N} ; \mathrm{C}_{\mathrm{p} 2}-\mathrm{C}$ atoms in the pyrrole rings bonded to $\mathrm{C}_{\mathrm{p} 1} ; \mathrm{C}_{\mathrm{ml}}-\mathrm{C}$ atoms in the methyl groups $\left(\mathrm{ZrO}(\mathrm{OMP})\right.$ ); $\mathrm{C}_{\mathrm{b} 1}-\mathrm{C}$ atoms in the benzene rings bonded to $\mathrm{C}_{\mathrm{p} 2}(\mathrm{ZrOPc}) ; \mathrm{C}_{\mathrm{b} 2}-\mathrm{C}$ atoms in the benzene rings bonded to $\mathrm{C}_{\mathrm{b} 1}(\mathrm{ZrOPc})$. $\mathrm{H}$ atoms have the same indices as the $\mathrm{C}$ atoms that they are bonded.

${ }^{\mathrm{b}} \mathrm{C}_{\mathrm{m}}$ in $\mathrm{ZrOP}$ and $\mathrm{ZrO}(\mathrm{OMP}), \mathrm{N}_{\mathrm{m}}$ in $\mathrm{ZrOPz}$ and $\mathrm{ZrOPc}$. 
Table 2. Excited states of oxozirconium porphyrin and octamethylporphyrin (with excitation energies less than $4 \mathrm{eV}$ for the singlet states). Data for similar oxotitanium complexes ${ }^{[3]}$ are given for comparison.

\begin{tabular}{|c|c|c|c|c|c|c|c|c|c|c|c|c|c|}
\hline & $E, \mathrm{eV}$ & $\lambda, \mathrm{nm}$ & $f^{\text {a }}$ & & $E, \mathrm{eV}$ & $\lambda, \mathrm{nm}$ & & $E, \mathrm{eV}$ & $\lambda, \mathrm{nm}$ & $f^{\text {a }}$ & & $E, \mathrm{eV}$ & $\lambda, \mathrm{nm}$ \\
\hline \multicolumn{7}{|c|}{ Oxozirconium porphyrin } & \multicolumn{7}{|c|}{ Oxotitanium porphyrin ${ }^{[3]}$} \\
\hline${ }^{1} \mathrm{E}$ & 2.337 & 530 & $5 \cdot 10^{-5}$ & ${ }^{3} \mathrm{E}$ & 1.680 & 738 & ${ }^{1} \mathrm{E}$ & 2.386 & 520 & 0.003 & ${ }^{3} \mathrm{E}$ & 1.745 & 711 \\
\hline${ }^{1} \mathrm{E}$ & 3.224 & 385 & 0.798 & ${ }^{3} \mathrm{E}$ & 2.018 & 614 & ${ }^{1} \mathrm{~B}_{2}$ & 2.960 & 419 & 0 & ${ }^{3} \mathrm{E}$ & 2.040 & 608 \\
\hline${ }^{1} \mathrm{~A}_{2}$ & 3.323 & 373 & 0 & ${ }^{3} \mathrm{~B}_{2}$ & 3.066 & 404 & ${ }^{1} \mathrm{~B}_{1}$ & 3.109 & 399 & 0 & ${ }^{3} \mathrm{~B}_{2}$ & 2.942 & 421 \\
\hline${ }^{1} \mathrm{~B}_{2}$ & 3.335 & 372 & 0 & ${ }^{3} \mathrm{~A}_{2}$ & 3.109 & 399 & ${ }^{1} \mathrm{E}$ & 3.402 & 364 & 1.610 & ${ }^{3} \mathrm{~B}_{1}$ & 3.095 & 401 \\
\hline${ }^{1} \mathrm{~B}_{1}$ & 3.361 & 369 & 0 & ${ }^{3} \mathrm{E}$ & 3.152 & 393 & ${ }^{1} \mathrm{~A}_{2}$ & 3.620 & 342 & 0 & ${ }^{3} \mathrm{~B}_{2}$ & 3.132 & 421 \\
\hline${ }^{1} \mathrm{~A}_{1}$ & 3.414 & 363 & 0.003 & ${ }^{3} \mathrm{~A}_{1}$ & 3.179 & 390 & & & & & & & \\
\hline${ }^{1} \mathrm{E}$ & 3.723 & 333 & 1.217 & ${ }^{3} \mathrm{~B}_{1}$ & 3.231 & 384 & & & & & & & \\
\hline${ }^{1} \mathrm{E}$ & 3.729 & 333 & 0.024 & ${ }^{3} \mathrm{~B}_{2}$ & 3.335 & 372 & & & & & & & \\
\hline${ }^{1} \mathrm{~B}_{2}$ & 3.801 & 326 & 0 & ${ }^{3} \mathrm{E}$ & 3.420 & 362 & & & & & & & \\
\hline${ }^{1} \mathrm{~A}_{2}$ & 3.891 & 319 & 0 & ${ }^{3} \mathrm{~B}_{2}$ & 3.561 & 348 & & & & & & & \\
\hline${ }^{1} \mathrm{~B}_{2}$ & 3.910 & 317 & 0 & ${ }^{3} \mathrm{~A}_{2}$ & 3.573 & 347 & & & & & & & \\
\hline \multicolumn{7}{|c|}{ Oxozirconium octamethylporphyrin } & \multicolumn{7}{|c|}{ Oxotitanium octamethylporphyrin ${ }^{[3]}$} \\
\hline${ }^{1} \mathrm{E}$ & 2.293 & 541 & 0.018 & ${ }^{3} \mathrm{E}$ & 1.690 & 734 & ${ }^{1} \mathrm{E}$ & 2.327 & 533 & 0.033 & ${ }^{3} \mathrm{E}$ & 1.715 & 723 \\
\hline${ }^{1} \mathrm{E}$ & 3.135 & 395 & 0.855 & ${ }^{3} \mathrm{E}$ & 1.961 & 632 & ${ }^{1} \mathrm{~B}_{1}$ & 2.902 & 427 & 0 & ${ }^{3} \mathrm{E}$ & 2.019 & 614 \\
\hline${ }^{1} \mathrm{~A}_{2}$ & 3.269 & 379 & 0 & ${ }^{3} \mathrm{~B}_{2}$ & 2.875 & 431 & ${ }^{1} \mathrm{~B}_{2}$ & 2.997 & 414 & 0 & ${ }^{3} \mathrm{~B}_{1}$ & 2.888 & 429 \\
\hline${ }^{1} \mathrm{~B}_{2}$ & 3.278 & 378 & 0 & ${ }^{3} \mathrm{~A}_{2}$ & 2.899 & 428 & ${ }^{1} \mathrm{E}$ & 3.289 & 377 & 1.499 & ${ }^{3} \mathrm{~B}_{2}$ & 2.909 & 426 \\
\hline${ }^{1} \mathrm{~B}_{1}$ & 3.344 & 371 & 0 & ${ }^{3} \mathrm{E}$ & 2.913 & 426 & ${ }^{1} \mathrm{~A}_{2}$ & 3.381 & 366 & 0 & ${ }^{3} \mathrm{~A}_{2}$ & 2.938 & 422 \\
\hline${ }^{1} \mathrm{~A}_{1}$ & 3.403 & 364 & 0.004 & ${ }^{3} \mathrm{~A}_{1}$ & 3.063 & 405 & ${ }^{1} \mathrm{~B}_{2}$ & 3.392 & 366 & 0 & ${ }^{3} \mathrm{~B}_{2}$ & 2.977 & 416 \\
\hline${ }^{1} \mathrm{E}$ & 3.424 & 362 & 0.017 & ${ }^{3} \mathrm{~B}_{1}$ & 3.113 & 398 & ${ }^{1} \mathrm{E}$ & 3.481 & 356 & 0.144 & ${ }^{3} \mathrm{E}$ & 2.992 & 414 \\
\hline${ }^{1} \mathrm{E}$ & 3.617 & 343 & 1.459 & ${ }^{3} \mathrm{E}$ & 3.233 & 383 & ${ }^{1} \mathrm{~B}_{1}$ & 3.501 & 354 & 0 & ${ }^{3} \mathrm{~A}_{1}$ & 3.098 & 400 \\
\hline${ }^{1} \mathrm{~B}_{2}$ & 3.711 & 334 & 0 & ${ }^{3} \mathrm{~B}_{2}$ & 3.421 & 362 & ${ }^{1} \mathrm{~A}_{1}$ & 3.582 & 346 & 0.002 & ${ }^{3} \mathrm{~B}_{1}$ & 3.166 & 392 \\
\hline${ }^{1} \mathrm{~A}_{2}$ & 3.713 & 334 & 0 & ${ }^{3} \mathrm{~B}_{1}$ & 3.476 & 357 & & & & & & & \\
\hline${ }^{1} \mathrm{~B}_{1}$ & 3.767 & 329 & 0 & ${ }^{3} \mathrm{~B}_{2}$ & 3.564 & 349 & & & & & & & \\
\hline${ }^{1} \mathrm{~A}_{1}$ & 3.800 & 326 & $3 \cdot 10^{-5}$ & ${ }^{3} \mathrm{~A}_{2}$ & 3.569 & 347 & & & & & & & \\
\hline${ }^{1} \mathrm{~B}_{2}$ & 3.807 & 326 & 0 & ${ }^{3} \mathrm{~A}_{1}$ & 3.591 & 345 & & & & & & & \\
\hline${ }^{1} \mathrm{~B}_{1}$ & 3.837 & 323 & 0 & ${ }^{3} \mathrm{~B}_{1}$ & 3.619 & 343 & & & & & & & \\
\hline
\end{tabular}

a Oscillator strength, multiplied by 2 for doubly-degenerate ${ }^{1} \mathrm{E}$ states.

As in the Ti species, there is clear correlation between the size of the coordination cavity and the pyramid height, the larger the cavity, the lower the pyramid. The changes of all other parameters are within $0.003 \AA$ for distances and 0.1 degrees for angles, being probably too small for discussing them.

Vibrational modes of the studied oxozirconium complexes can be classified among the symmetry species as follows:

$$
\begin{aligned}
& \Gamma=16 \mathrm{~A}_{1}+11 \mathrm{~A}_{2}+13 \mathrm{~B}_{1}+14 \mathrm{~B}_{2}+27 \mathrm{E}(\mathrm{ZrOP}) \\
& \Gamma=14 \mathrm{~A}_{1}+10 \mathrm{~A}_{2}+12 \mathrm{~B}_{1}+12 \mathrm{~B}_{2}+24 \mathrm{E}(\mathrm{ZrOPz}) \\
& \Gamma=5 \mathrm{~A}_{1}+20 \mathrm{~A}_{2}+22 \mathrm{~B}_{1}+23 \mathrm{~B}_{2}+45 \mathrm{E}(\mathrm{ZrO}(\mathrm{OMP})) \\
& \Gamma=23 \mathrm{~A}_{1}+19 \mathrm{~A}_{2}+21 \mathrm{~B}_{1}+21 \mathrm{~B}_{2}+42 \mathrm{E}(\mathrm{ZrOPc})
\end{aligned}
$$

This labeling corresponds to the orientation of the molecules in such a way that the $\mathrm{Zr}-\mathrm{N}$ bonds are located in $x z$ and $y z$ planes. Modes of $\mathrm{A}_{1}$ and $\mathrm{E}$ symmetries are active in the infrared spectra. The calculated infrared spectra of four oxotitanium complexes are presented in Figure 5 (Tables of numerical values of frequencies and intensities are available as Supplementary information.) In order to simulate the shape of a typical experimental spectrum the individual bands were described by Lorentz curves with a half-width of $10 \mathrm{~cm}^{-1}$.

Excited states of the four studied macroheterocyclic complexes (with the excitation energies less than $4 \mathrm{eV}$ ) are listed in Tables 2 and 3; data for oxotitanium complexes from study ${ }^{[3]}$ are given for comparison. It is interesting to note that the lowest excited states in all molecules are very similar to ones in the corresponding oxotitanium complexes (see ref. ${ }^{[3]}$ ). All of them are ${ }^{1} \mathrm{E}$ states and the corresponding wavelengths differ by no more than $10 \mathrm{~nm}$ from those in the TiO complexes. Oxozirconium phthalocyanine is especially demonstrative: the calculated wavelength of its $Q$ band is only $1 \mathrm{~nm}$ larger than in oxotitanium phthalocyanine ${ }^{[3]}$ (618 vs. $617 \mathrm{~nm}$ ). Hence, it may be suggested that the nature of the central ion has very little influence on the first excited electronic states of these molecules in their isolated states.

The Soret band in ZrO porphyrin and octamethylporphyrin complexes is bathochromically shifted by $c a .20 \mathrm{~nm}$ in comparison to oxotitanium species (from 364 to $385 \mathrm{~nm}$ in porphyrin and from 377 to $395 \mathrm{~nm}$ in octamethylporphyrin). The visible part of the phthalocyanine spectrum remains 
Table 3. Excited states of oxozirconium porphyrazine and phthalocyanine (with excitation energies less than $4 \mathrm{eV}$ for the singlet states). Data for similar oxotitanium complexes ${ }^{[3]}$ are given for comparison.

\begin{tabular}{|c|c|c|c|c|c|c|c|c|c|c|c|c|c|}
\hline & $E, \mathrm{eV}$ & $\lambda, \mathrm{nm}$ & $f^{\mathrm{a}}$ & & $E, \mathrm{eV}$ & $\lambda, \mathrm{nm}$ & & & & & & & \\
\hline \multicolumn{7}{|c|}{ Oxozirconium porphyrazine } & \multicolumn{7}{|c|}{ Oxotitanium porphyrazine ${ }^{[3]}$} \\
\hline${ }^{1} \mathrm{E}$ & 2.394 & 517 & 0.229 & ${ }^{3} \mathrm{E}$ & 1.440 & 861 & ${ }^{1} \mathrm{E}$ & 2.429 & 510 & 0.291 & ${ }^{3} \mathrm{E}$ & 1.450 & 855 \\
\hline${ }^{1} \mathrm{E}$ & 2.842 & 436 & 0.145 & ${ }^{3} \mathrm{E}$ & 2.440 & 508 & ${ }^{1} \mathrm{~A}_{2}$ & 3.228 & 384 & 0 & ${ }^{3} \mathrm{E}$ & 2.621 & 473 \\
\hline${ }^{1} \mathrm{~A}_{2}$ & 2.856 & 434 & 0 & ${ }^{3} \mathrm{~B}_{2}$ & 2.684 & 462 & ${ }^{1} \mathrm{~B}_{1}$ & 3.231 & 384 & 0 & ${ }^{3} \mathrm{~B}_{2}$ & 2.752 & 451 \\
\hline${ }^{1} \mathrm{~B}_{2}$ & 2.865 & 433 & 0 & ${ }^{3} \mathrm{~A}_{2}$ & 2.698 & 459 & ${ }^{1} \mathrm{~B}_{2}$ & 3.250 & 381 & 0 & ${ }^{3} \mathrm{~A}_{2}$ & 2.768 & 448 \\
\hline${ }^{1} \mathrm{~B}_{1}$ & 2.889 & 429 & 0 & ${ }^{3} \mathrm{E}$ & 2.713 & 457 & ${ }^{1} \mathrm{E}$ & 3.312 & 374 & $2 \cdot 10^{-5}$ & ${ }^{3} \mathrm{E}$ & 2.860 & 434 \\
\hline${ }^{1} \mathrm{~A}_{1}$ & 2.934 & 423 & 0.003 & ${ }^{3} \mathrm{~A}_{1}$ & 2.801 & 443 & ${ }^{1} \mathrm{E}$ & 3.329 & 372 & 0.045 & ${ }^{3} \mathrm{~A}_{1}$ & 3.037 & 408 \\
\hline${ }^{1} \mathrm{E}$ & 3.221 & 384 & $8 \cdot 10^{-4}$ & ${ }^{3} \mathrm{~B}_{1}$ & 2.824 & 439 & ${ }^{1} \mathrm{~B}_{1}$ & 3.358 & 369 & 0 & ${ }^{3} \mathrm{E}$ & 3.066 & 404 \\
\hline${ }^{1} \mathrm{E}$ & 3.284 & 378 & 0.008 & ${ }^{3} \mathrm{E}$ & 2.974 & 417 & ${ }^{1} \mathrm{~A}_{1}$ & 3.452 & 359 & 0.003 & ${ }^{3} \mathrm{~B}_{1}$ & 3.095 & 401 \\
\hline${ }^{1} \mathrm{~A}_{2}$ & 3.475 & 356 & 0 & ${ }^{3} \mathrm{~A}_{2}$ & 3.054 & 406 & ${ }^{1} \mathrm{E}$ & 3.555 & 349 & 0.207 & ${ }^{3} \mathrm{~B}_{1}$ & 3.210 & 386 \\
\hline${ }^{1} \mathrm{~B}_{2}$ & 3.479 & 356 & 0 & ${ }^{3} \mathrm{E}$ & 3.133 & 396 & & & & & & & \\
\hline${ }^{1} \mathrm{~A}_{1}$ & 3.576 & 347 & 0.004 & ${ }^{3} \mathrm{~A}_{1}$ & 3.172 & 391 & & & & & & & \\
\hline${ }^{1} \mathrm{~B}_{1}$ & 3.586 & 345 & 0 & ${ }^{3} \mathrm{~B}_{2}$ & 3.270 & 379 & & & & & & & \\
\hline${ }^{1} \mathrm{~B}_{2}$ & 3.634 & 341 & 0 & ${ }^{3} \mathrm{~A}_{1}$ & 3.323 & 373 & & & & & & & \\
\hline${ }^{1} \mathrm{~A}_{2}$ & 3.677 & 337 & 0 & ${ }^{3} \mathrm{~B}_{1}$ & 3.337 & 372 & & & & & & & \\
\hline${ }^{1} \mathrm{~B}_{1}$ & 3.703 & 335 & 0 & ${ }^{3} \mathrm{~B}_{1}$ & 3.345 & 371 & & & & & & & \\
\hline${ }^{1} \mathrm{~A}_{1}$ & 3.705 & 335 & $2 \cdot 10^{-4}$ & ${ }^{3} \mathrm{~A}_{2}$ & 3.612 & 343 & & & & & & & \\
\hline${ }^{1} \mathrm{E}$ & 3.940 & 314 & 1.054 & ${ }^{3} \mathrm{~B}_{1}$ & 3.622 & 342 & & & & & & & \\
\hline${ }^{1} \mathrm{~B}_{1}$ & 3.958 & 313 & 0 & ${ }^{3} \mathrm{E}$ & 3.650 & 340 & & & & & & & \\
\hline \multicolumn{7}{|c|}{ Oxozirconium phthalocyanine } & \multicolumn{7}{|c|}{ Oxotitanium phthalocyanine ${ }^{[3]}$} \\
\hline${ }^{1} \mathrm{E}$ & 2.007 & 618 & 0.774 & ${ }^{3} \mathrm{E}$ & 1.063 & 1167 & ${ }^{1} \mathrm{E}$ & 2.009 & 617 & 0.779 & ${ }^{3} \mathrm{E}$ & 1.069 & 1160 \\
\hline${ }^{1} \mathrm{E}$ & 3.100 & 400 & 0.081 & ${ }^{3} \mathrm{E}$ & 2.761 & 449 & ${ }^{1} \mathrm{~B}_{1}$ & 2.433 & 510 & 0 & ${ }^{3} \mathrm{~B}_{1}$ & 2.419 & 513 \\
\hline${ }^{1} \mathrm{~A}_{2}$ & 3.139 & 394 & 0 & ${ }^{3} \mathrm{~B}_{2}$ & 2.841 & 436 & ${ }^{1} \mathrm{~B}_{2}$ & 3.264 & 380 & 0 & ${ }^{3} \mathrm{~B}_{2}$ & 2.848 & 435 \\
\hline${ }^{1} \mathrm{~B}_{2}$ & 3.144 & 394 & 0 & ${ }^{3} \mathrm{E}$ & 2.887 & 429 & ${ }^{1} \mathrm{~A}_{2}$ & 3.265 & 380 & 0 & ${ }^{3} \mathrm{E}$ & 2.862 & 433 \\
\hline${ }^{1} \mathrm{~B}_{1}$ & 3.228 & 384 & 0 & ${ }^{3} \mathrm{~A}_{2}$ & 2.894 & 428 & ${ }^{1} \mathrm{E}$ & 3.311 & 375 & 0.013 & ${ }^{3} \mathrm{~A}_{2}$ & 2.923 & 424 \\
\hline${ }^{1} \mathrm{~B}_{1}$ & 3.203 & 387 & 0 & ${ }^{3} \mathrm{~B}_{1}$ & 2.972 & 417 & ${ }^{1} \mathrm{~B}_{1}$ & 3.377 & 367 & 0 & ${ }^{3} \mathrm{~B}_{1}$ & 2.999 & 413 \\
\hline${ }^{1} \mathrm{~A}_{1}$ & 3.270 & 379 & 0.004 & ${ }^{3} \mathrm{~A}_{1}$ & 3.010 & 412 & ${ }^{1} \mathrm{~B}_{2}$ & 3.418 & 363 & 0 & ${ }^{3} \mathrm{~A}_{1}$ & 3.064 & 405 \\
\hline${ }^{1} \mathrm{E}$ & 3.289 & 377 & $4 \cdot 10^{-6}$ & ${ }^{3} \mathrm{~B}_{2}$ & 3.066 & 404 & ${ }^{1} \mathrm{~B}_{1}$ & 3.465 & 358 & 0 & ${ }^{3} \mathrm{~B}_{2}$ & 3.066 & 404 \\
\hline${ }^{1} \mathrm{E}$ & 3.398 & 365 & 0.006 & ${ }^{3} \mathrm{~B}_{1}$ & 3.073 & 403 & ${ }^{1} \mathrm{~A}_{1}$ & 3.475 & 357 & 0.002 & ${ }^{3} \mathrm{E}$ & 3.071 & 404 \\
\hline${ }^{1} \mathrm{~B}_{2}$ & 3.441 & 360 & 0 & ${ }^{3} \mathrm{E}$ & 3.193 & 388 & ${ }^{1} \mathrm{E}$ & 3.499 & 354 & 0.005 & ${ }^{3} \mathrm{~B}_{1}$ & 3.145 & 394 \\
\hline${ }^{1} \mathrm{~A}_{2}$ & 3.462 & 358 & 0 & ${ }^{3} \mathrm{E}$ & 3.209 & 386 & ${ }^{1} \mathrm{~A}_{2}$ & 3.554 & 349 & 0 & ${ }^{3} \mathrm{~A}_{2}$ & 3.249 & 382 \\
\hline${ }^{1} \mathrm{~B}_{2}$ & 3.497 & 355 & 0 & ${ }^{3} \mathrm{~A}_{2}$ & 3.229 & 384 & ${ }^{1} \mathrm{E}$ & 3.576 & 347 & 0.238 & ${ }^{3} \mathrm{E}$ & 3.283 & 378 \\
\hline${ }^{1} \mathrm{~A}_{1}$ & 3.525 & 352 & $2 \cdot 10^{-5}$ & ${ }^{3} \mathrm{~B}_{1}$ & 3.234 & 383 & & & & & & & \\
\hline${ }^{1} \mathrm{~A}_{2}$ & 3.533 & 351 & 0 & ${ }^{3} \mathrm{~A}_{1}$ & 3.240 & 383 & & & & & & & \\
\hline${ }^{1} \mathrm{~B}_{1}$ & 3.551 & 349 & 0 & ${ }^{3} \mathrm{~B}_{2}$ & 3.295 & 376 & & & & & & & \\
\hline${ }^{1} \mathrm{E}$ & 3.588 & 346 & 0.855 & ${ }^{3} \mathrm{E}$ & 3.339 & 371 & & & & & & & \\
\hline${ }^{1} \mathrm{~B}_{2}$ & 3.639 & 341 & 0 & ${ }^{3} \mathrm{~B}_{1}$ & 3.346 & 371 & & & & & & & \\
\hline${ }^{1} \mathrm{E}$ & 3.638 & 341 & 0.463 & ${ }^{3} \mathrm{~A}_{2}$ & 3.376 & 367 & & & & & & & \\
\hline${ }^{1} \mathrm{~A}_{1}$ & 3.708 & 334 & 0.004 & ${ }^{3} \mathrm{~A}_{1}$ & 3.388 & 366 & & & & & & & \\
\hline${ }^{1} \mathrm{~B}_{1}$ & 3.715 & 334 & 0 & ${ }^{3} \mathrm{~B}_{2}$ & 3.409 & 364 & & & & & & & \\
\hline${ }^{1} \mathrm{~A}_{2}$ & 3.753 & 330 & 0 & ${ }^{3} \mathrm{~B}_{1}$ & 3.411 & 363 & & & & & & & \\
\hline${ }^{1} \mathrm{~A}_{2}$ & 3.858 & 321 & 0 & ${ }^{3} \mathrm{E}$ & 3.508 & 353 & & & & & & & \\
\hline${ }^{1} \mathrm{~B}_{1}$ & 3.869 & 320 & 0 & ${ }^{3} \mathrm{~A}_{2}$ & 3.542 & 350 & & & & & & & \\
\hline${ }^{1} \mathrm{E}$ & 3.876 & 320 & 0.118 & ${ }^{3} \mathrm{~A}_{1}$ & 3.566 & 348 & & & & & & & \\
\hline${ }^{1} \mathrm{~A}_{1}$ & 3.888 & 319 & 0.002 & ${ }^{3} \mathrm{~B}_{2}$ & 3.603 & 344 & & & & & & & \\
\hline${ }^{1} \mathrm{~B}_{2}$ & 3.892 & 319 & 0 & ${ }^{3} \mathrm{~A}_{2}$ & 3.790 & 327 & & & & & & & \\
\hline
\end{tabular}

a Oscillator strength, multiplied by 2 for doubly-degenerate ${ }^{1} \mathrm{E}$ states. 


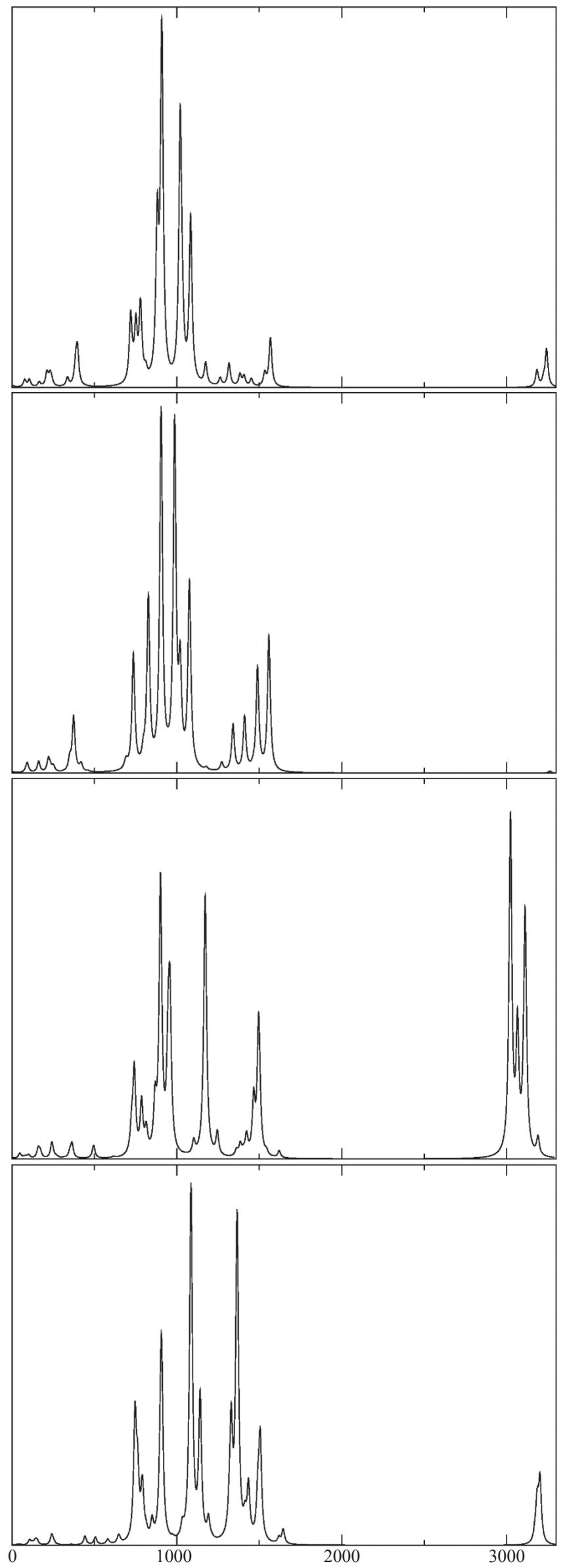

almost unchanged. On the other hand, in the visible spectrum of oxozirconium porphyrazine an absorption band at $436 \mathrm{~nm}$ is present, that has no analogue in the oxotitanium complex. The corresponding ${ }^{1} \mathrm{E}$ excited state in $\mathrm{ZrOPz}$ has a significant contribution of the HOMO- $\rightarrow$ LUMO +1 excitation (with the weight of 0.73 ). In turn, the HOMO-1 orbital is composed partially of $d$-type AOs of zirconium. Therefore, the oxozirconium porphyrazine represent the case of a very significant influence of the central ion on the visible electronic spectrum.

Acknowledgements. This work has been supported by a Russian Foundation for Basic Research (RFBR) grant no. 10-03-00884a. The author is grateful to Prof. Mikhail K. Islyaikin and to Prof. Georgiy V. Girichev for suggestions and discussion.

\section{References}

1. Hiller W., Strähle J., Kobel W., Hanack M. Z. Kristallogr. 1982, 159, 173-183.

2. Zakharov A.V., Shlykov S.A., Zhabanov Yu.A., Girichev G.V. Phys. Chem. Chem. Phys. 2009, 3472-3477.

3. Zakharov A.V., Girichev G.V. J. Mol. Struct.: THEOCHEM 2008, 851, 183-196.

4. Sakurai Y., Shiozaki H., Yokoyama M. J. Mol. Struct.: THEOCHEM 2006, 766, 41-47.

5. Kim H.J., Kim K. Acta Crystallogr., Sect. C: Cryst. Struct. Commun. 1999, 55, 1814-1815.

6. Brand H., Arnold J. Organometallics 1993, 12, 3655-3665.

7. Brand H., Arnold J. Angew. Chem., Int. Ed. 1994, 33, 95-97.

8. Brand H., Arnold J. J. Am. Chem. Soc. 1992, 114, 2266-2267.

9. Granovsky A.A. PC GAMESS version 7.0, http://classic.chem. msu.su/gran/gamess/index.html.

10. Schmidt M.W., Baldridge K.K., Boatz J.A., Elbert S.T., Gordon M.S., Jensen J.J., Koseki S., Matsunaga N., Nguyen K.A., Su S., Windus T.L., Dupuis M., Montgomery J.A. J. Comput. Chem. 1993, 14, 1347-1363.

11. Andrae D., Haeussermann U., Dolg M., Stoll H., Preuss H. Theoret. Chim. Acta 1990, 77, 123-141.

12. Martin J.M.L, Sundermann A. J. Chem. Phys. 2001, 114, 34083420.

13. Dunning T.H. J. Chem. Phys. 1971, 55, 716-723.

14. Urban M., Kellö V., Čársky P. Theoret. Chim. Acta 1977, 45, 205-213.

15. Hariharan P.C., Pople J.A. Theoret. Chim. Acta 1973, 28, 213222.

16. Strenalyuk T., Samdal S., Volden H.V. J. Phys. Chem., A 2007, 111, 12011-12018.

17. Samdal S., Volden H.V., Ferro V.R., García de la Vega J. M., González-Rodríguez D., Torres T. J. Phys. Chem., A 2007, 111, 4542-4550.

18. Zakharov A.V., Shlykov S.A., Bumbina N.V., Danilova E.A., Krasnov A.V., Islyaikin M.K., Girichev G.V. Chem. Commun. 2008, 3573-3575.

19. Zakharov A.V., Shlykov S.A., Danilova E.A., Krasnov A.V., Islyaikin M.K., Girichev G.V. Phys. Chem. Chem. Phys. 2009, $11,8570-8579$.

20. Danilova E.A., Melenchuk T.V., Trukhina O.N., Zakharov A.V., Islyaikin M.K. Macroheterocycles 2010, 3, 33-37.

21. Bode B.M., Gordon M.S. J. Mol. Graphics Mod. 1998, 16, 133-138.

Figure 5. Calculated infrared spectra of $\mathrm{ZrOP}, \mathrm{ZrOPz}, \mathrm{ZrO}(\mathrm{OMP})$, and $\mathrm{ZrOPc}$ (top to bottom, frequencies in $\mathrm{cm}^{-1}$, intensities in arbitrary units). 